\title{
Minimal important differences of EORTC QLQ-C30 for metastatic breast cancer patients: Results from a randomized clinical trial
}

\author{
Takuya Kawahara $^{1}$ (1) $\cdot$ Naruto Taira $^{2} \cdot$ Takeru Shiroiwa $^{3} \cdot$ Yasuhiro Hagiwara $^{4} \cdot$ Takashi Fukuda $^{3} \cdot$ Yukari Uemura $^{5}$. \\ Hirofumi Mukai ${ }^{6}$
}

Accepted: 19 December 2021 / Published online: 4 January 2022

(c) The Author(s) 2022

\begin{abstract}
Purpose To establish minimal important differences (MIDs) for the European Organisation for Research and Treatment for Cancer Quality of life Questionnaire core 30 (EORTC QLQ-C30) in patients with metastatic breast cancer.

Methods The dataset was obtained from the SELECT BC-CONFIRM randomized clinical trial. Anchors obtained from patients (transition items) and clinicians (performance status) were used for anchor-based methods. Anchors obtained through 6 months after starting treatment were used for this analysis. Correlation coefficients of anchor and change in QLQ-C30 and effect size were used to qualify for estimating MIDs. Mean change method and generalized estimating equation were applied to estimate MIDs. Distribution-based methods were used for comparison.

Results We analyzed a dataset of 154 metastatic breast cancer patients. MIDs were estimated in 8 of 15 scales of QLQ-C30. Estimated MIDs for within-group improvement varied from 7 to 15 and those for deterioration varied from -7 to -17 . Estimated MIDs for between-group improvement varied from 5 to 11 and those for deterioration varied from -5 to -8 across QLQ-C30 scales. Patient-reported anchors were more susceptible to early changes in health status than clinicianreported anchors.

Conclusion We provided the MIDs of the QLQ-C30 using both patient- and clinicians-reported anchors measured in a randomized trial of Japanese patients with metastatic breast cancer. We recommend patient-reported anchors for anchor-based estimation of MID. Our results can aid patients and clinicians, as well as researchers, in the interpretation of QLQ-C30.
\end{abstract}

Keywords Anchoring $\cdot$ EORTC QLQ-C30 $\cdot$ Health-related quality of life $\cdot$ Minimal important difference $\cdot$ Patient-reported outcomes

Takuya Kawahara

takuyakawahara@g.ecc.u-tokyo.ac.jp

1 Clinical Research Promotion Center, The University of Tokyo Hospital, 7-3-1 Hongo, Bunkyo-ku, Tokyo 113-8655, Japan

2 Breast and Endocrine Surgery Department, Okayama University Hospital, Okayama, Japan

3 Center for Outcomes Research and Economic Evaluation for Health, National Institute of Public Health, Wako, Japan

4 Department of Biostatistics, Division of Health Sciences and Nursing, The University of Tokyo, Tokyo, Japan

5 Department of Data Science, Center for Clinical Sciences, National Center for Global Health and Medicine, Tokyo, Japan

6 Department of Breast and Medical Oncology, National Cancer Center Hospital East, Kashiwa, Japan

\section{Introduction}

Treatment for metastatic breast cancer aims to prolong survival and palliate symptoms [1]. Considering that metastatic breast cancer remains incurable, maintaining quality of life (QOL) is an important therapeutic goal [2]. Instruments to measure QOL in patients with metastatic breast cancer include the European Organisation for Research and Treatment of Cancer Quality of Life Questionnaire core 30 (EORTC QLQ-C30). Despite improvements in validation of the QLQ-C30, interpreting the numerical scores remains challenging.

A large clinical trial can show statistically significant differences for QOL scores, but the clinical relevance of these differences remains controversial. The concept of minimal important difference (MID) provides a measure of the smallest difference in QOL scores that patients can 
perceive as improvement or deterioration [3]. King has comprehensively reviewed the literature on this topic [4]. While both anchor- and distribution-based methods are available for estimating MIDs, the anchor-based method has the appeal of incorporating external instruments (criterion or anchor) that are relevant to patients [5].

Recommendations [6] or guidelines across cancer sites using meta-analysis $[7,8]$ have been provided to interpret QLQ-C30. These are useful for designing trials and interpretation, but MIDs can vary according to factors such as patient populations, cancer site, and disease stage. Research into MIDs for metastatic breast cancer remains limited. Musoro et al. recently used two published trials to estimate MIDs relying on clinical anchors from clinician examinations [9]. In contrast, the most widely used anchor is the rating of change in health status as obtained directly from patients [10].

The purpose of this study was to determine the MIDs of the QLQ-C30 in a population of metastatic breast cancer patients using data from the SELECT BC-CONFIRM trial. In that trial, transition items reported directly from patients and other clinical anchors were collected at multiple timepoints from the initiation of treatment; these trial data were thus considered desirable for the estimation of MID.

\section{Methods}

\section{Patient population}

The dataset for this study comes from the SELECT BCCONFIRM randomized clinical trial, which enrolled and randomized 230 patients from multiple centers in Japan [11]. The trial aimed to confirm that S-1, an oral fluorouracil antitumor drug, is non-inferior to anthracycline-containing regimens as first-line chemotherapy for HER2-negative metastatic breast cancer. Patients were randomized to receive oral S-1 or intravenous anthracycline (doxorubicin/ cyclophosphamide or epirubicin/cyclophosphamide) at a standard dose. The main inclusion criteria for the trial were as follows: presence of HER2-negative metastatic breast cancer; endocrine therapy-resistant status; and no previous administration of chemotherapy for advanced disease. The primary endpoint in that trial was overall survival, and QOL was included as a secondary endpoint. The research ethics boards of all participating institutions approved this study, and all participants provided written informed consent prior to enrollment. The trial was registered with the University Hospital Medical Information Network, Japan (UMIN000005449).

\section{The EORTC QLQ-C30}

The EORTC QLQ-C30 is a general QOL instrument for cancer patients [12]. This questionnaire comprises 30 items, 24 of which are aggregated into five functional scales (physical, role, emotional, cognitive, and social), three symptom scales (fatigue, pain, and nausea/vomiting), and one global health status. The remaining six items assess additional symptoms (dyspnea, appetite loss, insomnia, constipation, and diarrhea) and financial impact. The SELECT BC-CONFIRM trial used the Japanese version of the QLQ-C30 questionnaire (version 3) [13], with each scale/item converted into a score ranging from 0 to 100. A high score in the functional scale represents increased functional ability, whereas a high score in the symptom scale represents increased distress. Researchers administered the QLQ-C30 before starting the protocol treatment and every 2 months thereafter until 1 year after starting treatment.

\section{Anchors}

We selected two anchors: the patient's global rating of transition; and change in Eastern Cooperative Oncology Group performance status (PS) [14]. The transition items comprised three questions: "Compared with when you started the chemotherapy in this trial: (a) How has the time you spend in bed or a chair during the day changed? (a question of function); (b) How have your concerns changed? (a question of concerns); and (c) How has your global health status changed? (a question of global health)". We created the transition items for this study by referring to the item representativeness of each functional scale of the QLQ-C30 using factor analysis [15] of a previous clinical trial in Japan [16]. For each question, seven response options were provided, such as "much shorter", "shorter", "a little shorter", "unchanged", "a little longer", "longer", or "much longer" for the functional question. We devised the 7-grade responses based on the previous study [6]. In the trial, researchers administered the transition questionnaire with the QLQ-C30 every 2 months until 6 months after starting the protocol treatment. Researchers scored PS between 0 (fully active) to 4 (completely disabled) at every course of the treatment.

Three status groups were defined for transition items. At each timepoint, patients who made a response adjacent to "unchanged" were categorized as deterioration or improvement. Patients who responded two categories away from "unchanged" were excluded from analysis for estimation of MIDs, as these responses were considered beyond a "minimal" change. For example, patients who answered 
"a little longer", "unchanged", or "a little shorter" for the functional question were categorized as showing "deterioration", "stable (no change)", and "improvement", respectively, and all other responses were excluded from the analysis of that timepoint. Similarly, at each timepoint, three change status groups were defined for the anchor of PS, calculated as the change in score from baseline: deterioration (worsened by one category of the anchor); stable (no change); or improvement (improved by one category of the anchor). Changes by two or more scores of PS were excluded from analysis, under the same rationale described above.

\section{Statistical analysis}

We summarized patient characteristics and demographics of the analyzed cohort. The variables included age, weight, number of lymph node metastases, estrogen receptor status, progesterone receptor status, history of surgery, history of chemotherapy use, and history of oral fluorouracil use.

We compared changes in QLQ-C30 scores with anchors measured at 2, 4, and 6 months from baseline. We calculated correlation coefficient $r$, and considered $r>|0.3|$ as necessary to show a moderate association between change scores and anchors [17]. We calculated Pearson's correlation, Spearman's rank correlation, polyserial correlation, and polychoric correlation. We compared the 0.3 -threshold and the maximum of the four correlation coefficients to explore candidate scales of QLQ-C30.

We used the mean change method to estimate MIDs for within-group change. The within-group MID can be calculated in several ways; some scholars calculate mean change in a (slightly) improved/deteriorated group [18], while other scholars adjust the results by subtracting the mean change that occurs in the stable group $[5,9,19,20]$. As the latter method has been more frequently applied in cancer research, we calculated the mean difference in change scores between deterioration and stable groups to indicate within-group MIDs for deterioration. Similarly, the mean difference of change scores between improvement and stable groups indicates MIDs for improvement. We calculated effect sizes (ESs) as the mean difference in change scores divided by the standard deviation (SD) of the QOL scale at baseline. ESs determine the responsiveness of changes in QOL to changes in anchor categories. We considered that $\mathrm{ESs} \geq 0.2$ and $<0.8$ were appropriate to include as MIDs because an ES $<0.2$ is small, and an ES $\geq 0.8$ is large [21]. We calculated withingroup MIDs separately for each pair of the anchor and timepoint (i.e., at 2, 4, and 6 months). To reduce the possibility of false-positive results, we show the results of QOL with at least an estimated two MIDs across timepoints or anchors.

We used a generalized estimating equation (GEE) to estimate MIDs for between-group change; we fitted the GEE for a given QOL and anchor pair, the changes in QOL over multiple timepoints as outcome variables, and anchor category as dummy explanatory variables. In contrast to the mean change method, the GEE method stacks changes in QOL scores over the timepoints and treats the scores as a vector of outcome. In comparative clinical trials in which patients respond to the QOL questionnaire longitudinally, the GEE with changes in QOL over multiple timepoints as outcome variables and treatment variable as explanatory variables is often fitted, rather than the simple linear regression which only uses a change in QOL at a specific timepoint. The GEE method thus provides a useful MID for between-group change [9]. We also applied the threshold for ESs $(\geq 0.2$ and $<0.8)$.

We obtained final estimates of MIDs provided by anchorbased methods using the correlation-based weighted average to reflect the degree of closeness of each pair of the anchor and change in QOL.

We also conducted a distribution-based method for MIDs. We calculated the $0.2 \mathrm{SD}, 0.3 \mathrm{SD}, 0.5 \mathrm{SD}$, and standard error of measurement based on a test-retest reliability of [22] for each QOL at all timepoints.

We conducted all statistical analyses using Base SAS and SAS/STAT version 9.4 software of the SAS System for Windows (SAS Institute, Cary, NC, USA).

\section{Results}

In total, 230 patients were randomized in the trial. To calculate the change in QOL from baseline, we included only the 154 patients who had completed a baseline QLQ-C30. Table 1 summarizes the demographic and treatment characteristics of the analyzed cohort. Median age was about 60 years. The majority of patients had a history of surgery (76.6\%), and about two-thirds of patients had no history of chemotherapy.

Numbers of patients by anchor categories and corresponding mean change in QOL scores are shown in Supplementary Table 1 . When using the transition item of global health, the proportions of "improvement", "stable (no change)", and "deterioration" were 17\%, 38\%, and $23 \%$, respectively, with the remaining $22 \%$ responding two or more categories away from "stable (no change)". When using the change in PS anchor, the proportions of "improvement", "stable (no change)", and "deterioration" were $6 \%, 78 \%$, and $13 \%$, respectively. Table 2 shows correlation coefficients between change in QLQ-C30 and anchors. For each anchor, correlated QOL scales (i.e., maximum correlation coefficient $>0.3$ ) at one or more timepoints are listed. The performance status showed a correlation with change in QLQ-C30, mostly at 6 months. Supplementary Table 2 shows correlation coefficients for 
Table 1 Patient characteristics

\begin{tabular}{|c|c|c|c|}
\hline & Anthracycline $(n=74)$ & $\mathrm{S}-1(n=80)$ & Total $(n=154)$ \\
\hline Age (years), median (IQR) & $60.5(52-66)$ & $59.5(49-64)$ & $60.0(50-65)$ \\
\hline Weight $(\mathrm{kg})$, median (IQR) & $52.0(48-59)$ & $53.0(48-60)$ & $52.0(48-60)$ \\
\hline \multicolumn{4}{|l|}{ Performance status } \\
\hline 0 & $61(82.4)$ & $64(80.0)$ & $125(81.2)$ \\
\hline 1 & $13(17.6)$ & $16(20.0)$ & $29(18.8)$ \\
\hline \multicolumn{4}{|c|}{ Number of lymph node metastases } \\
\hline 0 & $24(32.4)$ & $20(25.0)$ & $44(28.6)$ \\
\hline $1-3$ & $17(23.0)$ & $27(33.8)$ & $44(28.6)$ \\
\hline $4-9$ & $11(14.9)$ & $6(7.5)$ & $17(11.0)$ \\
\hline$>9$ & $5(6.8)$ & $7(8.8)$ & $12(7.8)$ \\
\hline Unknown & $17(23.0)$ & $20(25.0)$ & $37(24.0)$ \\
\hline \multicolumn{4}{|l|}{ Estrogen receptor } \\
\hline+ & $56(75.7)$ & $62(77.5)$ & $118(76.6)$ \\
\hline- & $17(23.0)$ & $11(13.8)$ & $28(18.2)$ \\
\hline Unknown & $1(1.4)$ & $7(8.8)$ & $8(5.2)$ \\
\hline \multicolumn{4}{|l|}{ Progesterone receptor } \\
\hline+ & $45(60.8)$ & $52(65.0)$ & $97(63.0)$ \\
\hline- & $26(35.1)$ & $21(26.3)$ & $47(30.5)$ \\
\hline Unknown & $3(4.1)$ & $7(8.8)$ & $10(6.5)$ \\
\hline \multicolumn{4}{|l|}{ History of surgery } \\
\hline No & $18(24.3)$ & $18(22.5)$ & $36(23.4)$ \\
\hline Yes & $56(75.7)$ & $62(77.5)$ & $118(76.6)$ \\
\hline \multicolumn{4}{|l|}{ History of chemotherapy } \\
\hline No & $49(66.2)$ & $57(71.3)$ & $106(68.8)$ \\
\hline Yes & $25(33.8)$ & $23(28.8)$ & $48(31.2)$ \\
\hline \multicolumn{4}{|l|}{ History of oral fluorouracil } \\
\hline No & $63(85.1)$ & $69(86.3)$ & $132(85.7)$ \\
\hline Yes & $11(14.9)$ & $11(13.8)$ & $22(14.3)$ \\
\hline
\end{tabular}

Data represent $n(\%)$ unless stated otherwise. IQR inter quartile range

absolute or baseline scores with anchors. In most pairs of anchors and scales, correlations of transition items with change scores were greater than correlations of transition items with absolute scores. In most pairs of positively (negatively) correlated transition items and scales, transition items were negatively (positively) correlated with the score at baseline.

Figure 1 shows the range of MIDs from the mean change method for each QOL scale across multiple anchors. The mean change method identified MIDs for improvement and deterioration in 8 of 15 QLQ-C30 scales, and the GEE identified MIDs for at least one direction in 7 of the 15 QLQ-C30 scales. Estimated MIDs differed between scales of QOL, direction (improvement and deterioration), and methods of estimation. Estimates from GEE tended to be smaller than those from the mean change method. No MIDs were estimated from GEE on some scales. Supplementary Table 3 shows details of results from Fig. 1. Most MIDs estimated with GEE were $p<0.05$, but this was not the case for MIDs estimated under the mean change method.
Table 3 summarizes MIDs from anchor-based methods, alongside results from the distribution-based method for comparison. Distribution-based estimates at 2 months are presented in Table 3 because results at other timepoints were similar, with at most a difference of 0.8 units for 0.2 SD. Most MIDs for interpretation of within-group differences were between 0.3 and $0.5 \mathrm{SD}$.

\section{Discussion}

To the best of our knowledge, this study is the first to estimate MIDs of QLQ-C30 in a population of Japanese patients with metastatic breast cancer. The study identified MIDs for interpreting within-group differences in 8 scales and for interpreting between-group differences in 7 scales.

As far as we know, only one study has examined the MIDs of QLQ-C30 in patients with advanced breast cancer [9]. Musoro et al. used two published trials to identify MIDs [9], and most of the patients were from European 
Table 2 Correlations between changes in quality of life scales and anchors at 2, 4, 6 months from randomization

\begin{tabular}{|c|c|c|c|c|}
\hline Anchor & Scale & $\begin{array}{l}2 \text { months } \\
(n=125- \\
130)\end{array}$ & $\begin{array}{l}4 \text { months } \\
(n=116- \\
121)\end{array}$ & $\begin{array}{l}6 \text { months } \\
(n=103- \\
111)\end{array}$ \\
\hline \multirow[t]{10}{*}{ Function } & QL & 0.69 & 0.40 & 0.36 \\
\hline & $\mathrm{PF}$ & 0.40 & 0.42 & 0.44 \\
\hline & $\mathrm{RF}$ & 0.31 & 0.29 & 0.44 \\
\hline & $\mathrm{EF}$ & 0.17 & 0.34 & 0.34 \\
\hline & SF & 0.31 & 0.36 & 0.27 \\
\hline & FA & 0.34 & 0.49 & 0.34 \\
\hline & PA & 0.15 & 0.34 & 0.26 \\
\hline & DY & 0.35 & 0.16 & 0.15 \\
\hline & SL & 0.07 & 0.25 & 0.41 \\
\hline & $\mathrm{AP}$ & 0.39 & 0.24 & 0.17 \\
\hline \multirow[t]{6}{*}{ Concern } & QL & 0.33 & 0.36 & 0.30 \\
\hline & $\mathrm{PF}$ & 0.18 & 0.26 & 0.34 \\
\hline & $\mathrm{RF}$ & 0.05 & 0.14 & 0.36 \\
\hline & $\mathrm{EF}$ & 0.27 & 0.27 & 0.46 \\
\hline & SF & 0.21 & 0.40 & 0.34 \\
\hline & PA & 0.22 & 0.31 & 0.23 \\
\hline \multirow[t]{11}{*}{ Global health } & QL & 0.46 & 0.33 & 0.44 \\
\hline & $\mathrm{PF}$ & 0.39 & 0.31 & 0.41 \\
\hline & RF & 0.30 & 0.27 & 0.45 \\
\hline & $\mathrm{EF}$ & 0.22 & 0.23 & 0.44 \\
\hline & SF & 0.37 & 0.27 & 0.36 \\
\hline & FA & 0.37 & 0.24 & $\mathbf{0 . 3 3}$ \\
\hline & NV & 0.31 & 0.04 & 0.18 \\
\hline & PA & 0.35 & 0.31 & 0.37 \\
\hline & DY & 0.33 & 0.08 & 0.19 \\
\hline & SL & 0.06 & 0.12 & 0.33 \\
\hline & AP & 0.37 & 0.21 & 0.34 \\
\hline \multirow[t]{5}{*}{ Performance status } & QL & 0.14 & 0.38 & 0.25 \\
\hline & $\mathrm{PF}$ & 0.17 & 0.25 & 0.31 \\
\hline & RF & 0.20 & 0.17 & $\mathbf{0 . 3 0}$ \\
\hline & $\mathrm{CO}$ & 0.03 & 0.21 & 0.38 \\
\hline & DI & 0.08 & 0.16 & 0.30 \\
\hline
\end{tabular}

Because the number of patients varied by quality of life scale and anchor, $n$ is presented as a range for all scales and anchors. Correlations are shown in absolute values, with correlations $>|0.3|$ shown in bold

$Q L$ global quality of life; $P F$ physical function; $R F$ role function; $E F$ emotional function; $S F$ social function; $F A$ fatigue; $N V$ nausea and vomiting; $P A$ pain; $D Y$ dyspnea; $S L$ insomnia; $A P$ appetite loss; $C O$ constipation; $D I$ diarrhea

countries. They identified at least one suitable MID in eight scales, and six of those eight scales (global quality of life, physical function, role function, social function, fatigue, and appetite loss) were in common with the present findings. In these scales, MIDs for interpreting between-group were similar. Our results thus support their conclusions, at least for these scales.

Conversely, MIDs for interpreting within-group deterioration tended to be larger in our study than in the previous study [9], such as for global quality of life ( -10 vs. -8$)$, role function ( -17 vs. -6$)$, social function $(-12$ vs. -7$)$, and fatigue ( -10 vs. -8$)$. Feng et al. [23] investigated systematic differences between Japanese and European general populations in self-reported QOL questionnaires. They found that the percentage of Japanese respondents reporting no problems in morbidity, usual activities, pain, or anxiety was higher than that of European respondents [23]. Our study of Japanese patients revealed that minimally deteriorated patients described larger changes from baseline than the European patients reported by Musoro et al. [9]. These findings may indicate inter-country differences in the self-evaluation of health at a single point as well as changes between two timepoints, especially in terms of deterioration.

Consistent with recent findings on MIDs for other cancer sites [5, 19, 24, 25], the magnitudes of MIDs for deterioration and improvement differed, along with the scales of QLQ-C30. We observed no systematic differences between previous results and our findings. These estimates generally do not conflict with the previously proposed guidelines [7, 8], although we consider that MIDs for interpreting deteriorations and improvements should be distinguished. In addition, MIDs for interpreting within- and between-group changes differed, as shown in Table 3 . These results strongly indicate that MIDs fitted for purpose are necessary.

The choice of an anchor is one of the critical components in the estimation of anchor-based MIDs [10]. Musoro et al. [9] estimated MIDs relying on clinician-examined anchors such as performance status and common terminology criteria for adverse events. However, patient-reported anchors appear preferable to clinician-reported anchors [10]. The present study used both types of anchor: transition items and performance status. We found that transition items were more sensitive to early changes in QOL than performance status, as shown in Table 2. This finding is consistent with findings from previous research that self-reports from patients are more sensitive than clinicians' reports to underlying changes in health status, and tend to identify clinically meaningful symptoms earlier [26, 27]. MID estimates by mean change methods using performance status as the anchor lay within the range of MID estimates using transition items as the anchor (Supplementary Table 3). This finding may indicate that clinician-reported anchors provide similar results to patient-reported anchors for estimating MIDs. However, we recommend patient-reported anchors as more susceptible to changes in the underlying health status of patients than clinician-reported anchors. Note that one needs to be cautious about the use of transition items because they are often vulnerable to recall bias. 
Fig. 1 Minimal important differences obtained using the mean change method and generalized estimating equations. Symptom scales were reversed to align with functional scales; therefore, positive values represent decreases in symptom scales. Absence of the line (e.g., GEE estimates for deterioration in PF) indicates that no minimal important difference estimate that met our criteria was obtained. GEE generalized estimating equation; $Q L$ global quality of life; $P F$ physical function; $R F$ role function; $E F$ emotional function; $S F$ social function; $F A$ fatigue; $P A$ pain; $A P$ appetite loss

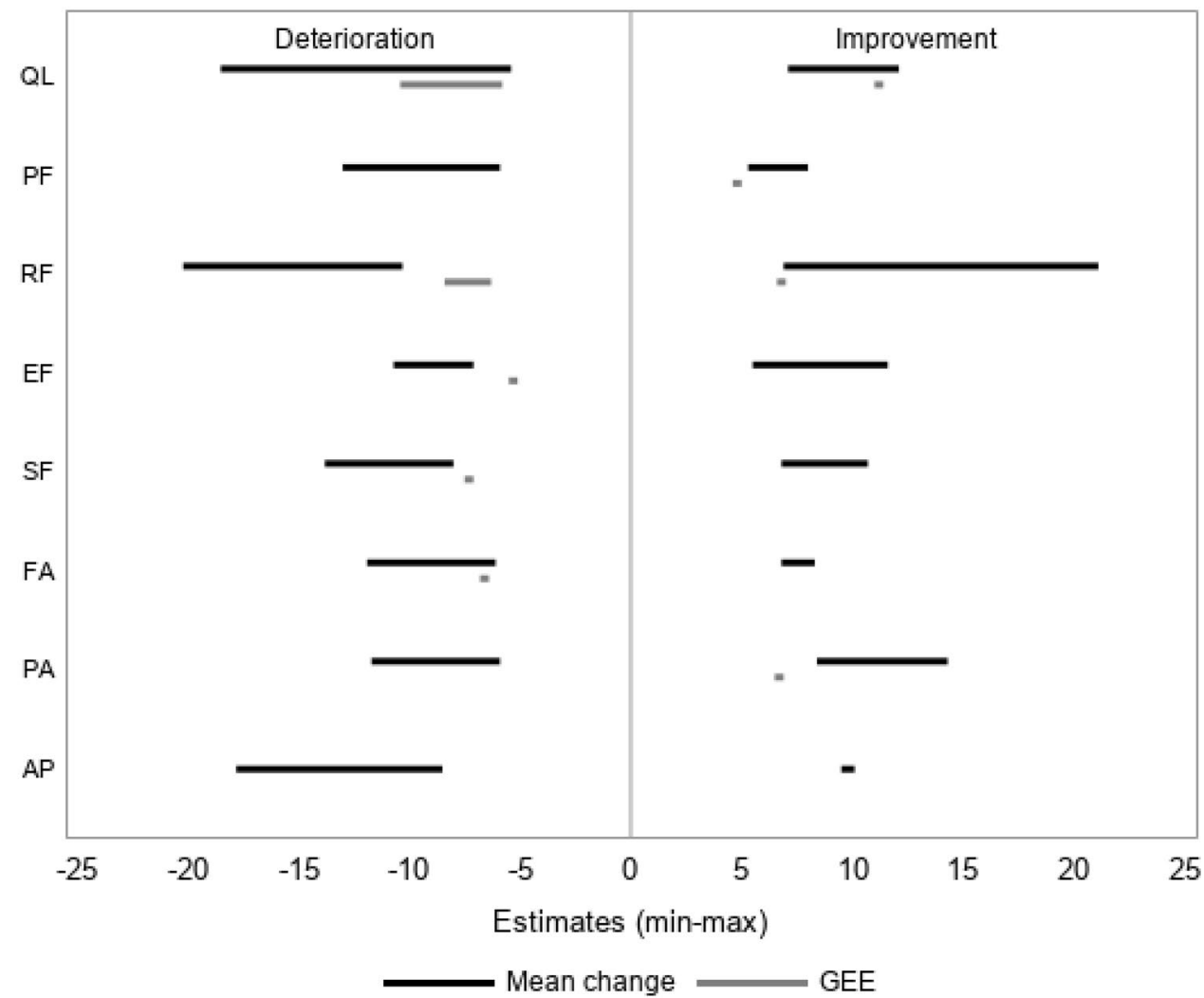

Table 3 Summary of minimal important differences from anchor- and distribution-based methods

\begin{tabular}{|c|c|c|c|c|c|c|c|c|}
\hline & \multicolumn{4}{|c|}{ Anchor-based method } & \multicolumn{4}{|c|}{ Distribution-based method } \\
\hline & \multicolumn{2}{|l|}{ Within-group } & \multicolumn{2}{|l|}{ Between-group } & \multirow[b]{2}{*}{$0.2 \mathrm{SD}$} & \multirow[b]{2}{*}{$0.3 \mathrm{SD}$} & \multirow[b]{2}{*}{$0.5 \mathrm{SD}$} & \multirow[b]{2}{*}{ SEM } \\
\hline & Improvement & Deterioration & Improvement & Deterioration & & & & \\
\hline QL & 9 & -10 & 11 & -8 & 4.4 & 6.6 & 11.0 & 9.3 \\
\hline $\mathrm{PF}$ & 7 & -9 & 5 & No MID & 3.7 & 5.6 & 9.3 & 5.6 \\
\hline $\mathrm{RF}$ & 15 & -17 & 7 & -7 & 5.5 & 8.2 & 13.7 & 11.6 \\
\hline $\mathrm{EF}$ & 9 & -7 & No MID & -5 & 4.0 & 6.0 & 10.0 & 7.5 \\
\hline SF & 9 & -12 & No MID & -7 & 4.7 & 7.1 & 11.8 & 8.5 \\
\hline FA & 7 & -10 & No MID & -7 & 4.3 & 6.5 & 10.8 & 8.9 \\
\hline PA & 11 & -11 & 7 & No MID & 4.7 & 7.0 & 11.7 & 8.8 \\
\hline AP & 10 & -9 & No MID & No MID & 5.9 & 8.8 & 14.7 & 13.5 \\
\hline
\end{tabular}

Symptom scales were reversed to align with functional scales; therefore, positive values represent decreases in symptom scales

$S E M$ standard error of measurement; $Q L$ global quality of life; $P F$ physical function; $R F$ role function; $E F$ emotional function; $S F$ social function; $F A$ fatigue; $P A$ pain; $A P$ appetite loss; $M I D$ minimal important difference
Distinguishing between unreliable and credible MIDs is essential for MIDs to help patients and clinicians in making decisions. One such method is referring to a recent guideline [10] and reporting the credibility level of the anchors used to estimate MID. We created Supplementary Table 2 for this purpose. We note here two points. First, the correlations of transition items with change score were greater than those with absolute score. Otherwise, the transition item seems to capture current health status more than the change in health status. Second, transition items showed some correlation not only to the change in QOL, but also to QOL at baseline. This correlation reflects the fact that patients appropriately respond to transition items while considering the health status at baseline. These points support the credibility of the transition items used in this study. 
Several limitations need to be considered when interpreting the present results. First, due to the small or medium sample size for estimating MID, each estimate was not precise. We aggregated estimates into a single MID using correlationweighted averages, so we consider our final estimates as reliable to some extent. Second, no change group or improved or deteriorated group showed statistically significant results in some scales. We do not believe, however, that this seriously influenced our conclusions, because all estimates of MIDs in this study exceeded effect size thresholds.

In conclusion, we provided the MIDs of QLQ-C30 using both patient- and clinician-reported anchors measured in a randomized trial of Japanese patients with metastatic breast cancer. Our results can aid patients and clinicians, as well as researchers, with interpreting QLQ-C30. Further studies should replicate this study in other populations or other anchors to validate the current MID estimates.

Supplementary Information The online version contains supplementary material available at https://doi.org/10.1007/s11136-021-03074-y.

Acknowledgements We thank all the patients and their families who participated in the trial and the clinical investigators involved in the trial.

Author contributions Conceptualization: TK, NT, TS, and YU; Data curation: HM; Formal analysis: TK; Funding acquisition: HM; Investigation: HM; Methodology: TK; Project administration: TK; Resources: HM; Software: TK; Supervision: NT; Validation: TK; Writing-original draft: TK; Writing_review \& editing: NT, TS, YH, TF, YU, and HM.

Funding This study was sponsored by the Comprehensive Support Project for Oncology Research (CSPOR) of the Public Health Research Foundation. Research funds were provided to CSPOR by Taiho Pharmaceutical Company Limited under the study contract. Taiho Pharmaceutical took no part in this study other than providing information relevant to proper use of the study drug.

Data availability The datasets generated and analyzed during the current study are available from the corresponding author on reasonable request.

Code availability The SAS codes generated during the current study are available from the corresponding author on reasonable request.

\section{Declarations}

Conflict of interest Dr. Mukai received honoraria from Pfizer, Takeda, Daiichi Sankyo and Taiho, and research grants from the Japanese government, Daiichi Sankyo, and Pfizer outside the submitted work. Dr. Mukai is a member of the Board of Directors of the Japan Breast Cancer Society.

Ethical approval The research ethics boards of all participating institutions approved the protocol of this study. This study was conducted according to the Declaration of Helsinki.

Informed consent All patients provided written informed consent.
Open Access This article is licensed under a Creative Commons Attribution 4.0 International License, which permits use, sharing, adaptation, distribution and reproduction in any medium or format, as long as you give appropriate credit to the original author(s) and the source, provide a link to the Creative Commons licence, and indicate if changes were made. The images or other third party material in this article are included in the article's Creative Commons licence, unless indicated otherwise in a credit line to the material. If material is not included in the article's Creative Commons licence and your intended use is not permitted by statutory regulation or exceeds the permitted use, you will need to obtain permission directly from the copyright holder. To view a copy of this licence, visit http://creativecommons.org/licenses/by/4.0/.

\section{References}

1. Waks, A. G., \& Winer, E. P. (2019). Breast cancer treatment: A review. Journal of the American Medical Association, 321, 288-300. https://doi.org/10.1001/jama.2018.19323

2. Harbeck, N., \& Gnant, M. (2017). Breast cancer. Lancet, 389, 1134-1150. https://doi.org/10.1016/S0140-6736(16)31891-8

3. Schünemann, H. J., \& Guyatt, G. H. (2005). Commentary-goodbye M(C)ID! Hello MID, where do you come from? Health Services Research, 40, 593-597. https://doi.org/10.1111/j.1475-6773. 2005.0k375.x

4. King, M. T. (2011). A point of minimal important difference. Expert Review of Pharmacoeconomics \& Outcomes Research, 11, 171-84. https://doi.org/10.1586/ERP.11.9.

5. Musoro, Z. J., Hamel, J. F., Ediebah, D. E., Cocks, K., King, M. T., Groenvold, M., et al. (2018). Establishing anchor-based minimally important differences (MID) with the EORTC qualityof-life measures: A meta-analysis protocol. BMJ Open, 8(1), 1-8. https://doi.org/10.1136/bmjopen-2017-019117.

6. Osoba, D., Rodrigues, G., Myles, J., Zee, B., \& Pater, J. (1998). Interpreting the significance of changes in health-related qualityof-life scores. Journal of Clinical Oncology, 16, 139-144. https:// doi.org/10.1200/JCO.1998.16.1.139

7. Cocks, K., King, M. T., Velikova, G., St-James, M. M., Fayers, P. M., \& Brown, J. M. (2011). Evidence-based guidelines for determination of sample size and interpretation of the European organisation for the research and treatment of cancer quality of life questionnaire core 30. Journal of Clinical Oncology, 29, 89-96. https://doi.org/10.1200/JCO.2010.28.0107

8. Cocks, K., King, M. T., Velikova, G., De Castro, G., Martyn StJames, M., Fayers, P. M., \& Brown, J. M. (2012). Evidence-based guidelines for interpreting change scores for the European organisation for the research and treatment of cancer quality of life questionnaire core 30. European Journal of Cancer, 48, 1713-1721. https://doi.org/10.1016/j.ejca.2012.02.059

9. Musoro, J. Z., Coens, C., Fiteni, F., Katarzyna, P., Cardoso, F., Russell, N. S., King, M. T., Cocks, K., Sprangers, M. A., Groenvold, M., \& Velikova, G. (2019). Minimally important differences for interpreting EORTC QLQ-C30 scores in patients with advanced breast cancer. JNCI Cancer Spectrum, 3, 1-7. https:// doi.org/10.1093/jncics/pkz037

10. Devji, T., Carrasco-Labra, A., Qasim, A., Phillips, M., Johnston, B. C., Devasenapathy, N., Zeraatkar, D., Bhatt, M., Jin, X., Brignardello-Petersen, R., \& Urquhart, O. (2020). Evaluating the credibility of anchor based estimates of minimal important differences for patient reported outcomes: Instrument development and reliability study. BMJ, $m 369,1714$. https://doi.org/10.1136/ bmj.m1714

11. Mukai, H., Uemura, Y., Akabane, H., Watanabe, T., Park, Y., Takahashi, M., et al. (2021). Anthracycline-containing regimens or taxane versus S-1 as first-line chemotherapy for metastatic 
breast cancer. British Journal of Cancer, 125(9), 1217-1225. https://doi.org/10.1038/s41416-021-01531-6.

12. Aaronson, N. K., Ahmedzai, S., Bergman, B., Bullinger, M., Cull, A., Duez, N. J., et al. (1993). The European organization for research and treatment of cancer QLQ-C30: a quality-of-life instrument for use in international clinical trials in oncology. Journal of the National Cancer Institute, 85(5), 365-376. https:// doi.org/10.1093/jnci/85.5.365.

13. Shimozuma, K., \& Eguchi, N. (2001). Development and clinical application of QOL measurement instruments for cancer patients: (I) Japanese versions of QOL questionnaires developed in North American or European countries and validation of reliability and validity of the FACT-B, a QOL question. Retrieved February 26, 2021, from https://www.jmari.med.or.jp/download/WP056.pdf.

14. Oken, M. M., Creech, R. H., \& Davis, T. E. (1982). Toxicology and response criteria of the Eastern Cooperative Oncology Group. American Journal of Clinical Oncology, 5, 649-655. https://doi. org/10.1097/00000421-198212000-00014.

15. McLachlan, S. A., Devins, G. M., \& Goodwin, P. J. (1999). Factor analysis of the psychosocial items of the EORTC QLQ-C30 in metastatic breast cancer patients participating in a psychosocial intervention study. Quality of Life Research, 8, 311-317. https:// doi.org/10.1023/A:1008939818096.

16. Watanabe, T., Sano, M., Takashima, S., Kitaya, T., Tokuda, Y., Yoshimoto, M., et al. (2009). Oral uracil and tegafur compared with classic cyclophosphamide, methotrexate, fluorouracil as postoperative chemotherapy in patients with node-negative, high-risk breast cancer: National surgical adjuvant study for breast cancer 01 trial. Journal of Clinical Oncology, 27(9), 1368-1374. https:// doi.org/10.1200/JCO.2008.18.3939.

17. Revicki, D., Hays, R. D., Cella, D., \& Sloan, J. (2008). Recommended methods for determining responsiveness and minimally important differences for patient-reported outcomes. Journal of Clinical Epidemiology, 61, 102-109. https://doi.org/10.1016/j. jclinepi.2007.03.012

18. Hays, R. D., Farivar, S. S., \& Liu, H. (2005). Approaches and recommendations for estimating minimally important differences for health-related quality of life measures. COPD: Jounal of Chronic Obstructive Pulmonary Disease, 2, 63-67. https://doi. org/10.1081/COPD-200050663

19. Maringwa, J. T., Quinten, C., King, M., Ringash, J., Osoba, D., Coens, C., Martinelli, F., Vercauteren, J., Cleeland, C. S., Flechtner, H., Gotay, C., Greimel, E., Taphoorn, M. J., Reeve, B. B., Schmucker-Von Koch, J., Weis, J., Smit, E. F., van Meerbeeck, J. P., \& Bottomley, A. (2011). Minimal important differences for interpreting health-related quality of life scores from the EORTC QLQ-C30 in lung cancer patients participating in randomized controlled trials. Supportive Care in Cancer, 19(11), 1753-1760. https://doi.org/10.1007/s00520-010-1016-5

20. Maringwa, J., Quinten, C., King, M., Ringash, J., Osoba, D., Coens, C., et al. (2011). Minimal clinically meaningful differences for the EORTC QLQ-C30 and EORTC QLQ-BN20 scales in brain cancer patients. Annals of Oncology, 22, 2107-2112. https://doi. org/10.1093/annonc/mdq726.

21. Cohen, J. (1988). Statistical power analysis for the behavioral sciences. Routledge. https://doi.org/10.4324/9780203771587.

22. Hjermstad, M. J., Fossa, S. D., Bjordal, K., \& Kaasa, S. (1995). Test/retest study of the European organization for research and treatment of cancer core quality-of-life questionnaire. Journal of Clinical Oncology, 13, 1249-1254. https://doi.org/10.1200/JCO. 1995.13.5.1249

23. Feng, Y., Herdman, M., van Nooten, F., Cleeland, C., Parkin, D., Ikeda, S., Igarashi, A., \& Devlin, N. J. (2017). An exploration of differences between Japan and two European countries in the self-reporting and valuation of pain and discomfort on the EQ-5D. Quality of Life Research, 26, 2067-2078. https://doi.org/10.1007/ s11136-017-1541-5

24. Hong, F., Bosco, J. L. F., Bush, N., \& Berry, D. L. (2013). Patient self-appraisal of change and minimal clinically important difference on the European organization for the research and treatment of cancer quality of life questionnaire core 30 before and during cancer therapy. BMC Cancer, 13, 1-8. https://doi.org/10.1186/ 1471-2407-13-165

25. Raman, S., Ding, K., Chow, E., Meyer, R. M., van der Linden, Y. M., Roos, D., et al. (2018). Minimal clinically important differences in the EORTC QLQ-C30 and brief pain inventory in patients undergoing re-irradiation for painful bone metastases. Quality of Life Research, 37, 1089-1098. https://doi.org/10.1007/ s11136-017-1745-8.

26. Basch, E., Jia, X., Heller, G., Barz, A., Sit, L., Fruscione, M., et al. (2009). Adverse symptom event reporting by patients vs clinicians: Relationships with clinical outcomes. Journal of National Cancer Institute, 101, 1624-1632. https://doi.org/10.1093/jnci/ djp386.

27. Basch, E. (2010). The missing voice of patients in drug-safety reporting. New England Journal of Medicine, 362, 865-869. https://doi.org/10.1056/NEJMp0911494

Publisher's Note Springer Nature remains neutral with regard to jurisdictional claims in published maps and institutional affiliations. 